\title{
Tea Table Mediator: A Multimodal Ambient Display on the Table Exploiting Five Senses Convergence
}

\author{
Hyun Sang Cho ${ }^{1}$, Kyoung Shin Park ${ }^{2}$, and Minsoo Hahn ${ }^{1}$ \\ ${ }^{1}$ Digital Media Laboratory, Information and Communications University, \\ 51-10 Dogok-dong, Gangnam-gu, Seoul 135-854, S. Korea \\ haemosulicu.ac.kr \\ ${ }^{2}$ Multimedia Engineering, Dankook University, \\ San 29 Anseo-dong, Cheonan-si, Chungnam, 330-714, Korea
}

\begin{abstract}
In the past, many tabletop systems have used the traditional table augmented with digital technologies to enhance collaborative works such as faceto-face or remote meeting and working. In this paper, we describe the Tea Table Mediator, a multimodal ambient tabletop display designed for five senses convergence experience combined with tea drinking. This ambient display enhances a traditional tea table with multimodal interfaces forintimate small group interaction. This paper describes the design considerations, the details on system implementation, and the discussion of a preliminary user evaluation.
\end{abstract}

Keywords: Tabletop Display, Sense Convergence, Small Group Interaction.

\section{Introduction}

People traditionally have used a table for formal face-to-face meetings or private social interactions (such as family gathering, tea party and games). Recently the tabletop systems became a emerging research field in collaborative technology with pervasive intelligent objects and tangible interface [2][5][7][8][11]. Some of them are specifically designed for supporting intimate or entertaining activities, such as digital photo album [6][10], game [5] and private discourses or tea party [9][11]. However, all these systems force users to share information on the table display with exposed technology and hence it is difficult to keep a privacy and interest of intimate personal communication.

In this paper, we present Tea Table Mediator, a new ambient display system that fosters intimate group communication at the tea table. It provides a private display on the surface of a tea and gives a five senses convergence experience to promote small group intimate interaction during a tea party. The tea party is a moment when people can have casual social interactions and one of group entertainments. Gaver suggested several design assumptions for ludic engagement suggested by the drift table design [11]: promoting curiosity, exploration and reflection, de-emphasizing the pursuit of external goals, and maintaining the openness and ambiguity. In the Tea Table 


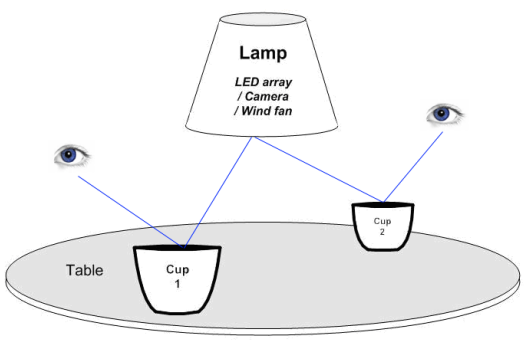

(a) Conceptual figure

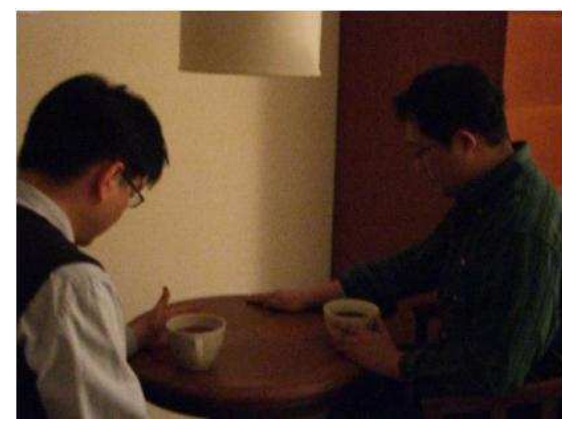

(b) Real System

Fig. 1. The current prototype of Tea Table Mediator

Mediator system, we provide an abstract form of images and sounds. This system allows users keep their intimacy without any disgust from exposure of technology. The system works with any cup, i.e., no special electric hardware components required on the cup. As shown in Fig. 1, the tea itself acts as a private display for images from a lamp above the table, which is shared within a group around a table.

By drinking tea people can relax and feel they are sharing time with others [3] and there have been several tabletop systems designed for tea [5][11]. However, they were limited to a simple tangible cup interface with additional electric components on the cup or makers. In these systems, more than two senses (such as, visual-auditory, visual-touch or visual-auditory-touch) are combined to make more efficient user experience [4][8] with traditional cup and table. Recently there is a study on emphasizing holistic sense convergence to promote effective user communication [1]. Nonetheless, it is hardly found the systems that support smell and taste senses. Tea itself gives the sense of taste and smells which is difficult to represent with current technologies. Fig. 2 shows the relationship of five senses user interaction provided by the Tea Table Mediator system. In addition, we intended to expand individual sense experience to group experience by combining the dynamic images generated by the system and the static color on the surface of tea.

\section{Design and Implementation}

Single Display Groupware (SDG) has a great advantage - that is, it makes group members easily concentrate on group topics displayed on the shared display. In the Tea Table Mediator system, we wanted to provide the shared group display while maintaining the group's privacy even where they are in public place as like café or bar. The Tea Table Mediator system uses the surface of tea as a private group display. The concept of the display is based on the law of reflection; the angle of incidence equals the angle of reflection. The LED display patterns are shown on the cups of tea. The users (at the round table) can see the same display pattern at the same time. Theoretically, it works with the infinite numbers of cup on the table. We noticed that the 


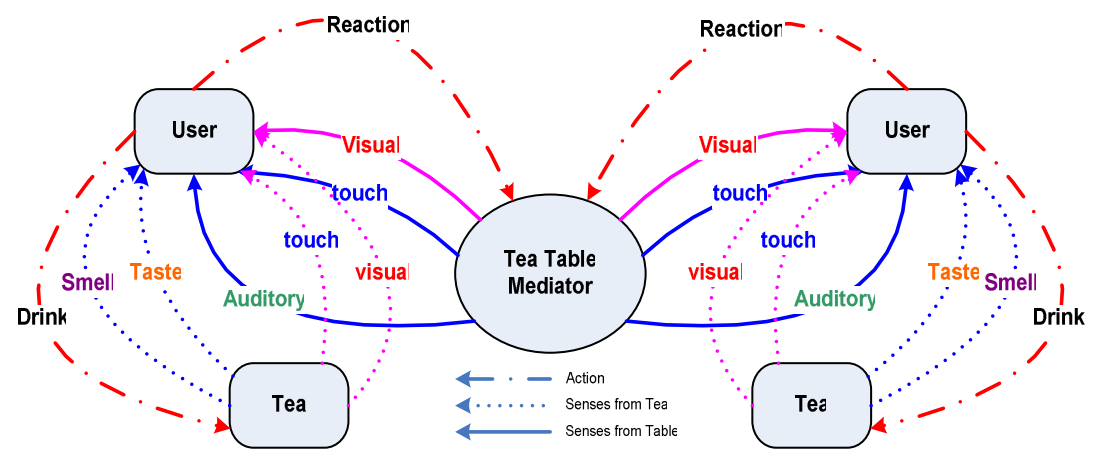

Fig. 2. The relation of five senses user interaction

tea also could satisfy some senses such as taste, smell and touch with temperature, which are difficult to serve conventional methods. The fusion of senses keeps users' interest.

For the intimate small group interaction such as friends and family, the privacy is a sensitive issue. Currently many tabletop systems display the information on the table surface. Such a public display disturbs people to keep concentration on their interaction topic and furthermore make people uncomfortable to use the system. It is a severe problem to play game or to enjoy entertaining rest with intimate members. While some applications served personalized display by mobile devices or directional view control film with multiple projectors [4][7]. The tea surface provides such private display and keeps the users' direction of sight on a virtual image in the cup to maintain users' concentration on their common topic. The holistic senses experience can enhance the emotional coziness.

Fig. 3 (a) shows the overall architecture of the Tea Table Mediator system. The system consists of a tea table with a lamp, and all the electric components are equipped in the lamp. In this system, any cup (preferably a large cup) can be used on the table and tea gives the private display surface for each user. Users can enjoy taste, smell and warm feeling while drinking tea. The system recognizes the kinds of tea that the user drinks (by detecting its color). It then displays additional visual, auditory and tactile patterns on the tea surface by the dynamic LED patterns and the wind generated by a fan embedded in the lamp. The LED patterns are reflected on the surface of tea in the cup. The lamp is composed of three main modules: the context recognition module, the interaction control module, and the display module. The main recognition module device is a camera module that detects the color of tea and the users' gesture. The camera continuously keeps watch over the cross-image marker area on the table. When a user places his/her cup on the marker, the changes of marker image is sent to a main PC. Then the image processing is performed by using the Video for Windows (VFW) library. It takes the $10 \times 10$ pixels marker-centered image as HSI data which is more robust for light intensity and the kinds of tea is classified by the data. 


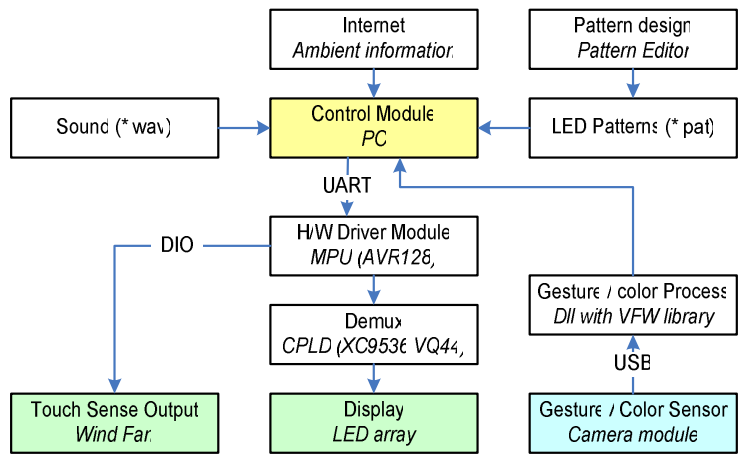

(a) System architecture

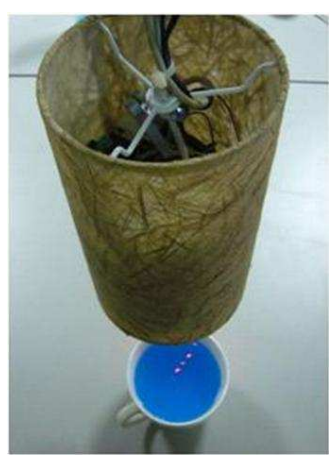

(b) Lamp

Fig. 3. The Architecture of the Tea Table Mediator

The interaction session started when the color of idle marker is changed. Once the interaction session started, the camera also detects the users' gesture. When the control module receives the change of the marker color, it combines the user's context (such as, the kinds of tea and the user's gestures) with the environmental context (such as, ambient temperature, outside weather condition, and current time and season) gotten from the internet to determine the interaction scenario. The scenario includes predefined LED patterns (written by a LED pattern editor), sound effects, and winds.

The display module consists of a $\mu$-controller hardware driver, a LED display array and a wind generation fan. Each LED on the array has three terminals for RGB colors. Fig. 3 (b) shows the structure of actual display module. The display module receives the interaction scenario from the control module through the RS-232C communication channel to the AVR128 micro controller. It sends the multiplexed LED pattern data to a demultiplex and latch module which consists of three XC9636 Xilinx Complex Programmable Logic Devices (CPLDs). Each CPLD was programmed by Verilog HDL as three-to-twenty four demultiplexer and latch. Users can see the reflected pattern of the LED array on the surface of tea. In addition, the micro controller directly operates a fan to generate ambient winds. The wind makes wave on the surface of tea to sway the reflected image on the surface of tea.

The first experience design was based on the tea colors. It consisted of simple scenarios, which gave different experiences by variation of host's sort (color) of tea. For example, when user put the green tea on the table, the green leave and moving caterpillar is reflected on the surface of tea. The LED patterns consisted of more than 15 images to show moving picture. Then the next experience included gesture detection. User could affect the image on LED array by their gesture to conduct simple game or interaction. They could push-pull their different colored area on the LED array. It could help to maintain and mediate their common attention. 


\section{User's Evaluation}

The Tea Table Mediator system has been installed in the ActiveHome, an actual home scale ubiquitous living environment testbed in Digital Media Lab, ICU, as shown in Fig. 1. Fig. 3 (b) shows the current prototype of the system.

The users reported the immersion of LED pattern image, which was formed beyond the tabletop. The image of reflected LED patterns formed beneath of a tea surface. Hence, it shows the effect that all users can see the LED display at the same position through their tea surfaces. It means that the reflected LED display operates as a single shared display beneath the tabletop that keeps all users' sight at the same position. They also reported that they felt intimacy among the members around table, which was shared only with the members around the table.

From this installation, we discovered the filtering effect caused by tea color. That is, the blue color LED patterns was washed out on the surface of blue tea. Through this filtering effect, we can make different images on the display surface for users who had different tea colors - especially, it works well with pure red, green, or blue beverage. We believe that it shows another interaction possibility to multiple users with the same LED pattern source.

The patterns were designed to present the abstractive expression (i.e., not clear shapes) to promote user's imagination and curiosity. This strategy was somewhat successful to attract some user's attention. The results showed careful pattern design is still important to attract users' interest consistently if the patterns are not definite but abstractive and ambiguous.

\section{Conclusion and Future Work}

In this paper, we presented the Tea Table Mediator, a multimodal ambient tabletop display system that provides private small group display and five senses convergence experience. The system combined a traditional tea and a digital tea table. While most previous works focused on the development of functionally augmented objects in the ubiquitous environment, our approach simply added digital expression with additional visual, auditory, and tactile feedback onto tea that provides smell and taste. The passive sense information (such as smell and taste) from tea was combined with the dynamic and active sense information from the tea table.

This ambient display is designed for enhancing a traditional tea table with multimodal interfaces for intimate small group interaction. The system detects the user context (such as, the color of tea that the user drinks) and then creates the dynamic LED patterns which is reflected onto the surface of tea. It also provides ambient sound and wind effects to enrich user's sensation. In this system, we suggested several sense fusion scenarios affected by user's interaction. For example, when a user drinks a green tea, he/she can see the green leaf and pine tree caterpillar LED patterns on the surface of tea combined with the bird chirp sounds and wind (the degree of wind blowing is affected by outside weather condition).

The results of user test showed the effectiveness of the Tea Table Mediator system. The users showed the interest to their experience with the system providing five senses expression. We found that the system provided a shared display to all users at 
the tea table. That is, all users could see the LED display images at virtually the same position beneath the table. We also found the LED pattern filtering effect caused by the tea color that can be used for the personalized information display. The low resolution of the LED array will be enhanced with high resolution and high intensity LCD.

We could confirmed the effectiveness of reflect type private display and holistic senses experience to attract and to keep small group users' interest from user evaluation and encouraged to apply this system to other apply this system to other tasks include not only intimate but also formal collaborative works. We are applying the personal reflective display to a tabletop type Computer Supported Collaborative Work (CSCW) based digital storytelling system and scenario that uses the social intelligence from the results of web information retrieval for educational field trip of students. We expect to evaluate the system performance for the suggested methods by traditional evaluation method of education. The system can be used for virtual tour conduction for tour club in public café.

\section{Acknowledgement}

This research was supported by the MIC (Ministry of Information and Communication), Korea, under the Digital Media Lab. support program supervised by the IITA (Institute of Information Technology Assessment).

\section{References}

1. Schmitt, B.H., Simonson, A.: Marketing Aesthetics: The Strategic Management of Branding, Identity and Image. Simon \& Schuster Inc., New York (1997)

2. Shen, C.: Multi-User Interface and Interactions on Direct-Touch Horizontal Surface: Collaborative Tabletop Research at MERL. In: TABLETOP'06 (2006)

3. Chung, H., Lee, C.-H.J., Selker, T.: Lover's Cups: Drinking Interfaces as New Communication Channels. In: CHI 2006 (April 2006)

4. Leikas, J., Strömberg, H., Ikonen, V., Suomela, R., Heinilä, J.: Multi-User Mobile Applications and a Public Display: Novel Ways for Social Interaction. In: PerCom 2006. 4th Pervasive Computing and Communications (2006)

5. Yoon, J., Oishi, J., Nawyn, J., Kobayashi, K., Gupta, N.: FishPong: Encouraging Humanto-Human Interaction in Informal Social Environments. In: CSCW'04 (November 2004)

6. Morris, M.R., Paepcke, A., Winograd, T., Stamberger, J.: TeamTag: Exploring Centralized versus Replicated Controls for Co-located Tabletop Groupware. In: CHI 2006 (April 2006)

7. Matsushita, M., Iida, M., Ohguro, T.: Lumisight Table: A Face-to-face Collaboration Support System That Optimizes Direction of Projected Information to Each Stakeholder. In: CSCW'04 (November 2004)

8. Ståhl, O., et al.: Information Exploration Using The Pond. In: CVE '02 (September 2002)

9. Stewart, J., Bederson, B., Druin: A Single display groupware: A model for co-present collaboration. In: CHI'99 (1999)

10. Apted, T., Kay, J., Quigley, A.: Tabletop Sharing of Digital Photographs for the Elderly. In: CHI 2006 (April 2006)

11. Gaver, W.W., et al.: The Drift Table: Designing for Ludic Engagement. In: CHI 2004 (April 2004) 